\section{Vowel and consonant clusters in word recognition}

\author{
CAROL MINNICK SANTA \\ and JOHN L. SANTA \\ Douglass College \\ Rutgers, The State University \\ New Brunswick, New Jersey 08903
}

Santa, Santa, and Smith (1977) have recently developed a reaction time task designed to explore the use of various letter clusters in word recognition. Basically, the technique was a same-different task wherein subjects saw a typed display consisting of a word followed by a word part or probe (BLAST:BL). The subject determined whether or not the probe was contained in the word. The probes included single letters, all combinations of adjacent double letters, triple letters, and whole words. On half of the trials, the probe matched a letter or letters in the word, and on half the trials, a single letter was changed so that the probe was not entirely contained in the word (BLAST:BLI). Using this technique, Santa et al. found evidence for a variety of subsyllable units in that the probes BL, BLA, AST, and BLAST were processed as quickly as the single-letter probes. We concluded that the accomplished reader is quite flexible in using a variety of processing units.

Santa (1976-1977) extended this work in an investigation of the development of word recognition units. Using this matching paradigm, she found that children seem to develop the ability to use different levels of analysis as they become more proficient readers. Remedial second graders were quite rigidly bound to single-letter analysis, while proficient second-grade readers were beginning to use subunit patterns such as the initial letter clusters. By fifth grade, students matched the adult pattern in their use of a greater variety of subword patterns.

There are, however, at least two difficulties with our program of research. First, we have explored a very limited set of word patterns; namely, words of the CCVCC (BLAST) and CCVCV (FRAME) forms. The second difficulty is that many of the patterns which appear to be processing units were also in advantageous serial positions within the word. For example, we found rapid processing of the BL and BLA in BLAST. Consequently, the results are somewhat contaminated by serial position effects. In the Santa et al. paper, we argued that a spelling pattern explanation was insufficient for explaining the entire

Send reprint requests to Carol M. Santa, Department of Education, Rutgers University, Douglass College, New Brunswick, New Jersey 08903. pattern of data. Furthermore, we conducted a control experiment using stimuli without vowels which contained either permissible or nonpermissible letter clusters (BLGST vs. TNGFP). The experiment revealed fairly substantial serial position effects such that initial consonant clusters were processed relatively quickly whether or not they formed legitimate letter clusters. However, we also obtained an interaction with permissibility such that permissible letter clusters were processed even more rapidly than nonpermissible consonant clusters.

The present experiment was designed to extend the class of word patterns we have investigated and to provide a more rigorous test of the serial position explanation. We employed words conforming to three patterns: CCVCC (BLAST), CCVVC (TREAT), and CVVCC (FAINT). According to a serial position explanation, the initial clusters (BL, TR, and FA) should behave similarly, but according to a spelling pattern explanation, the FA cluster in FAINT should be processed more slowly than the BL and TR clusters in BLAST and TREAT. In the former case, the FA would cross a hypothetical spelling pattern boundary and disrupt the medial vowel cluster, whereas, in the latter two examples, the spelling pattern would be preserved. Similarly, a serial position hypothesis predicts equal processing time for the medial clusters, but a spelling pattern interpretation predicts faster processing for probes preserving vowel clusters. Thus, one might expect the stimulus SHOUT:OU to be processed more quickly than FAINT:IN.

\section{METHOD}

\section{Subjects}

Sixteen college students participated in the experiment to fulfill a course requirement.

\section{Materials and Design}

The experimental task was a same-different procedure wherein subjects saw presentations of word-probe combinations. The stimuli consisted of a word, a colon (:), and a probe. The words were all five-letter English words following the CCVCC (BLAST), CCVVC (SHOUT), or CVVCC (FAINT) patterns.

The probe for each word-probe stimulus contained a letter pair taken from one of four possible positions in the word. For example, the probe could include the first letter doublet (SHOUT: SH), the second (SHOUT:HO), and third (SHOUT:OU), or the fourth letter pair (SHOUT:UT). For same trials, the probes matched an adjacent sequence of two letters in the word stimulus, but for different responses one letter was changed so that it did not match a letter sequence from the prior word stimulus (SHOUT:ET).

The stimulus set contained three lists of $\mathbf{8 0}$ words, one for each word type. For each word type, 40 words were used for same judgments and 40 for different judgments. Each of these 40 word sets were further divided so that there were stimuli representing each of the four different probe positions. For each word type, there were 10 words representing each of the four probes.

Finally, two presentation lists were formed. Each set of materials contained the same words, but the probe types assigned to 
each word differed across the two stimulus lists. Half of the 16 subjects saw List 1, while the remaining 8 subjects were presented List 2.

The final design was a 2 by 2 by 3 by 4 design with two lists, two levels of judgment, three word types, and four levels of probes. All factors were within variables except for $L$ ist, which was a between factor.

\section{Apparatus}

The experiment was controlled with an Altair computer with stimuli presented on 12 -in. Sony video monitors. The stimuli were presented in standard white on black, uppercase video characters produced by the Processor Technology VDM-1 video character generator. Subjects' responses and latencies in milliseconds were recorded on an ICOM floppy disk. A more complete description of the apparatus is available in Santa and Streit (1978). The stimuli were viewed from a distance of about $60 \mathrm{~cm}$ and subtended a visual angle of $2^{\circ} 26^{\prime}$ horizontally and $40^{\prime}$ vertically.

\section{Procedure}

Subjects were run in groups of four with stimuli presented to all four subjects for a period of $2 \mathrm{sec}$. The display was then terminated and followed by a 3-sec intertrial interval. The subjects were allowed to respond at any time while the stimulus was present or during the 3-sec intertrial interval.

All subjects were instructed on the nature of the matching task and then were told to press their dominant (right) hand button if the probe letters were fully contained in the word stimulus or their left hand button if one letter was different. They were encouraged to respond both as quickly and accurately as possible. After seeing several examples of the stimulus probe displays, the subjects were given a series of 16 practice trials. At the end of the practice trials, the experimenter asked if there were any questions and then proceeded to the 240-trial test sequence.

\section{RESULTS AND DISCUSSION}

Mean latencies for correct different judgments are shown in Table 1 as a function of word type and probe. However, we will focus on the analysis of same responses, since in this experiment, as in several previous ones (Egeth \& Blecker, 1971; Santa, Santa, \& Smith, 1977), the different judgments proved more variable and less sensitive to orthographic variations than the same judgments.

Figure 1 shows the mean reaction times for correct same judgments as a function of probe and word type. Errors were excluded from the analysis, since error rates were very low $(2.4 \%)$ and distributed randomly across conditions. For each subject, mean reaction times for same judgments were calculated for the four probes. An analysis of these data revealed a significant main effect of Probe $(F=20.24$, $\mathrm{df}=3 / 42, \mathrm{p}<.001)$ and a significant Word by Probe interaction $(F=17, \mathrm{df}=6 / 84, \mathrm{p}<.001)$.

Table 1

Mean Latencies (in Milliseconds) for Correct Different Judgments

\begin{tabular}{lllll}
\hline \multicolumn{1}{c}{ Word Type } & Probe 1 & Probe 2 & Probe 3 & Probe 4 \\
\hline CCVVC (SHOUT) & $1,269.6$ & $1,558.8$ & $1,306.3$ & $1,410.0$ \\
CVVCC (PAINT) & $1,307.6$ & $1,358.3$ & $1,347.4$ & $1,321.3$ \\
CCVCC (BLAST) & $1,393.8$ & $1,375.9$ & $1,334.2$ & $1,403.8$ \\
\hline
\end{tabular}

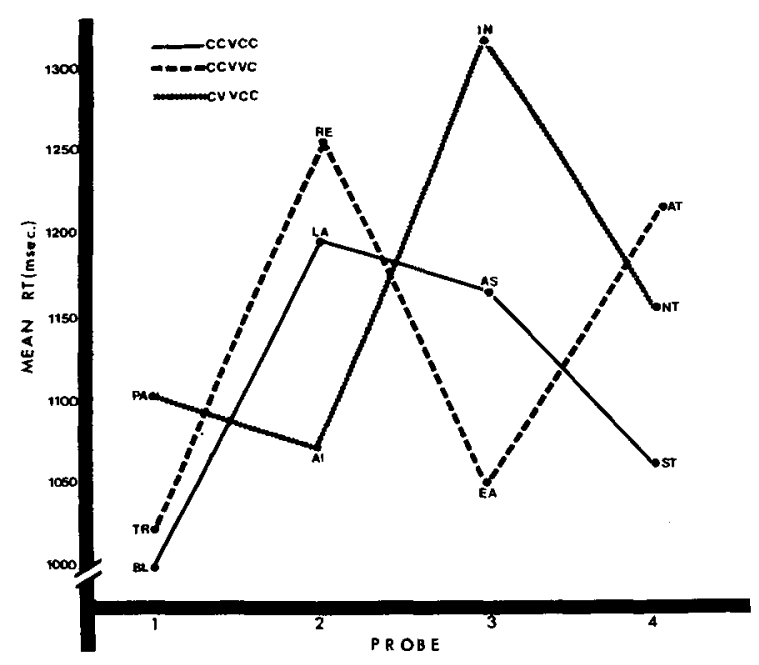

Figure 1. Mean latencies for correct same judgments as a function of probe and word type.

At first glance, the data displayed in Figure 1 seem to be arranged in an almost haphazard fashion. However, closer examination reveals that the reaction time patterns for each word type are quite predictable by a rudimentary notion of a spelling pattern. On the first probe, we see that initial consonant clusters are responded to very quickly as compared to the consonant-vowel probe in a word such as PAINT:PA $(p<.05) .{ }^{1}$ But, as we turn to the second and third probes, we see that a vowel cluster such as the AI in PAINT also appears as a functional unit of word processing as compared with probes which group together a consonant-vowel, for example, BLAST:LA, FLOAT:LO $(\mathrm{p}<.05)$.

In both the first and second probes, there is a clearly retarded latency whenever the probe breaks a natural consonant or vowel cluster. Moreover, the pattern of variation is quite unaccountable on a simple serial position explanation, since word pattern and not letter position seem to determine the speed of processing. Examination of the latencies for Probe 3 indicate that our paradigm is able to detect very subtle differences in the nature of the probes. On the third probe, there was nearly a $150-\mathrm{msec}$ difference separating each of the word types. As might be expected, the vowel cluster (TREAT:EA) was responded to most quickly. Of even more interest, there was a considerable difference between two seemingly identical probes, the AS in BLAST and the IN in PAINT. Both of these probes involve a vowelconsonant combination, but the IN in PAINT is responded to much more slowly than the AS in BLAST. The most reasonable explanation of this difference would seem to be that in one case (PAINT) the vowel is part of a larger unit (AI); but in the other case (BLAST), the vowel stands alone and is not so rigidly bound to consonants on either side. 
Finally, as we turn to the last probe, we see that the final consonant cluster in words such as BLAST and PAINT are responded to very quickly and the vowel-consonant combination of a word like TREAT: AT is responded to most slowly. For no apparent reason, the NT in a word like PAINT produced an intermediate latency. One would have more likely expected the NT of PAINT to be processed in the same fashion as the ST of BLAST. It might be noted that the usefulness on a final consonant cluster remains somewhat questionable. In the present experiment, we observe a discrepancy between the final consonant clusters of the PAINT and BLAST word types. Moreover, in our previous paper, Santa et al., the final consonant cluster was processed rapidly in some cases, but more often it did not seem to serve as a functional unit.

While the present paradigm has proved useful for investigating potential spelling patterns, some might be concerned that we are using a reaction time matching task to make claims about the word recognition process in reading. We are aware that a reaction time task such as ours might not involve the same processes as those used in reading. However, we do feel that the matching task has more commonalities to a reading situation than most laboratory paradigms. The natural reading situation does not involve matching two overtly presented stimulus strings, but it certainly does involve matching an encoded stimulus to preexisting representations of letter patterns.
Moreover, the context that is present in normal reading situations produces a task very much like our paradigm. That is, we often know what we will read before we actually see it, and in this sense reading becomes a simple pattern matching task without the trappings of a formal "yes-no" decision. Consequently, we feel that a matching paradigm affords a very reasonable method for investigating word recognition processes, and allows us to avoid the obvious difficulties of threshold presentations followed by recall or probed recognition.

\section{REFERENCES}

Egeth, H. E., \& Blecker, D. Differential effects of familiarity on judgments of sameness and differences. Perception \& Psychophysics, 1971, 9, 321-326.

SANTA, C. M. Spelling patterns and the development of flexible word recognition strategies. Reading Research Quarterly, 1976-1977, 12, 125-144.

Santa, J., Santa, C., \& Smith, E. Units of word recognition: Evidence for the use of multiple units. Perception \& Psychophysics, 1977, 22, 585-591.

SÁNTA, J., \& STREIr, P. A microprocessor reaction time laboratory. Behavior Research Methods \& Instrumentation, 1978, 10, 352-355.

\section{NOTE}

1. All differences among individual means reported in this paper have been with the Newman-Keuls procedure.

(Received and accepted for publication December 14, 1978.) 